\title{
WAVE HEIGHT DISTRIBUTION IN CONSTANT AND FINITE DEPTHS
}

\author{
Sofia Caires and Marcel R. A. van Gent ${ }^{1}$
}

\begin{abstract}
Several alternatives to the Rayleigh distribution have been proposed for describing individual wave heights in regions where depth-induced wave breaking occurs. The most widely used of these is the so-called Battjes and Groenendijk distribution. This distribution has been derived and validated in a context of a shallow water foreshore-waves propagating over a gently sloping shallow region towards the shore. Its validity for waves propagating in regions with shallow flat bottoms is investigated here. It is concluded that the distribution on average underestimates (outside its range of validity) high wave height measurements in shallow flat bottoms by as much as $15 \%$.
\end{abstract}

Keywords: Wave height distribution; flat bottoms; depth limited; flume data; field data; waves; shallow water

\section{INTRODUCTION}

In metocean studies the knowledge of the distribution of the individual wave heights in a sea state is often needed in order to estimate high quantiles up to the maximal wave height. In deep water the Rayleigh distribution is generally accepted as the wave height distribution. For finite depth and shallow regions various alternatives to the Rayleigh distribution have been proposed which account for depth-induced wave breaking, imposing thus restrictions on the distribution of higher individual wave heights in shallow waters. The most widely used depth-dependent distribution of the wave height is a composite Weibull distribution proposed by Battjes and Groenendijk (2000), hereafter referred to as the Battjes and Groenendijk distribution or the BG-distribution.

This distribution, as is the case for most of the available alternatives to the Rayleigh (Rattanapitikon, 2010), has been derived in a context of shallow water foreshores, i.e., when waves propagate over a sloping shallow region towards the coast. In fact, the BG-distribution was obtained from experimental data in flumes with foreshore slopes varying from 1:20 to 1:250 and its parameters depend on both the local water depth and slope. For a given water depth and significant wave height, the lower the slope, i.e. the flatter the bottom, the lower the high wave height quantiles of the BGdistribution. No data on waves propagating over flat bottoms have been used in the derivation and validation of the BG-distribution. In all tests on which the BG-distribution was based the sloping bathymetry causes continuing depth-induced wave breaking over the foreshore.

It is worth pointing out that on flat bottoms the ratio of significant wave height to depth (Van der Westhuysen, 2010) has been found to be higher than one would expect by decreasing the bottom slope in the available formulations for depth-induced wave breaking. This suggests that when waves propagate in constant depth regions the individual wave height distribution will deviate less from the Rayleigh distribution than the BG-distribution assuming a very flat slope. In order to avoid nonconservative/incautious designs it would therefore be of interest to study the distribution of individual wave heights propagating in constant depth regions.

This paper reports on a study aiming at investigating whether in regions of constant depth under depth-limited wave conditions the quantiles of the BG-distribution underestimate the high individual wave heights. The study is based on flume measurements carried for the purpose and on compiled existing field data.

The paper is organized as follows. We first provide an introduction to wave height distributions and describe the BG-distribution in detail, including an adjustment that needs to be done when implementing it so that the distribution converges to the Rayleigh in deep waters. Then we describe the measurements carried out in Deltares' Scheldt Flume and the compiled available field data from the lakes IJssel and Sloten in The Netherlands. Next we compare the measurements with the quantile estimates from the Rayleigh and the BG-distributions and discuss other alternatives. Finally, we provide conclusions and recommendations.

\section{WAVE HEIGTH DISTRIBUTION}

The distribution of an individual wave height $H$ in a sea state is usually described in terms of a cumulative distribution function, defined by $F(h)=P(H \leq h)$ for $h \geq 0$, where $P(E)$ denotes the

\footnotetext{
${ }^{1}$ Deltares, P.O. Box 177, 2600 MH Delft, The Netherlands; Sofia.Caires@deltares.nl
} 
probability of the event $E$ and $H \leq h$ is the event that a wave height $H$ is smaller than or equal to the numerical value $h$. Quantiles of the distribution of wave heights are computed in terms of $F$. By definition, the quantile/percentile of probability $1-1 / N$ of $F$ is the value of wave height that is exceeded on average once every $N$ waves. If we denote this value by $H_{1 / N}$ (or by $H_{100 \times 1 / N \%}$, if probabilities are expressed in percentages rather than proportions), we then have, by definition, that $N \times P\left(H>H_{1 / N}\right)=N \times\left[1-F\left(H_{1 / N}\right)\right]$, which is the expected number of exceedances of $H_{1 / N}$ among the $N$ waves, equals 1 . In other words, $H_{1 / N}$ is the solution to the equation $N\left[1-F\left(H_{1 / N}\right)\right]=1$ or $F\left(H_{1 / N}\right)=1-1 / N$.

A quantile that often needs to be computed in metocean studies is $H_{0.1 \%}$, the value of wave height that is exceeded on average once every 1,000 waves. However, data on individual wave heights from which the wave height distribution can be directly estimated are often unavailable. One therefore generally resorts to a standard parametric probability distribution as a model for wave height, whose parameters are determined by bottom information, when relevant, and sea-state integral wave parameters, such as significant wave height, which are more readily available from a wave model or from a statistical analysis of extremes of significant wave height (for instance the 100-yr return value of significant wave height).

\section{DEEP WATER REGIONS}

Longuet-Higgins (1952) proposed a Rayleigh distribution as a good model for the distribution of individual wave heights in sea-states governed by a narrow band energy spectrum in deep waters (see e.g. Goda, 1985, or Holthuijsen, 2007). Its corresponding distribution function is given by

$$
F(h)=1-\exp \left(-\left(\frac{h}{H_{r m s}}\right)^{2}\right), h>0
$$

where $H_{r m s}=\sqrt{8 m_{0}}$ is the root-mean-square wave height and $m_{0}$ is the variance of the sea surface or the integral (zero moment) of the (variance density) wave spectrum. This distribution is here referred to as the Rayleigh distribution. Note that, once $m_{0}$ is known, the Rayleigh distribution can be used to compute wave height quantiles as described above.

For historical reasons the sea state wave height parameter most commonly used is the mean height of the one-third highest waves, referred to as the significant wave height, $H_{s}$, which, according to the Rayleigh distribution, can be given by

$H_{s}=4 \sqrt{m_{0}}$.

In order to acknowledge that the significant wave height has been computed using (2), and not directly as the mean height of the one-third highest individual waves, $H_{s}$ is often denoted as $H_{m 0}$. In this study we shall only present estimates of $H_{m 0}$ and will always denote them by $H_{s}$. Note that the Rayleigh distribution can also be given in terms of $H_{s}$ :

$F(h)=1-\exp \left(-\left(\frac{h}{H_{s} / \sqrt{2}}\right)^{2}\right), h>0$.

Since the work of Longuet-Higgins several studies have shown that the Rayleigh distribution describes the distribution of individual wave height in deep waters reasonably well, even if the spectrum is not narrow-banded, and is therefore widely used.

\section{DEPTH LIMITED CONDITIONS}

For shallow waters various alternatives to the Rayleigh distribution have been proposed which account for depth-induced wave breaking, imposing thus restrictions on the distribution of higher individual wave heights in a shallow water sea state (see, for an overview, Rattanapitikon, 2010).

The most widely used shallow water distribution is the BG-distribution, which is described next.

Battjes and Groenendijk (2000) proposed a distribution of wave heights in shallow waters by a combination of two Weibull distributions, 
$F(h)= \begin{cases}1-\exp \left[-\left(\frac{h}{H_{1}}\right)^{k_{1}}\right] & h<H_{t r} \\ 1-\exp \left[-\left(\frac{h}{H_{2}}\right)^{k_{2}}\right] & h \geq H_{t r},\end{cases}$

with five unknown parameters, and which can be written in terms of normalized wave heights,

$\tilde{h}=h / H_{r m s}$,

as

$F(\tilde{h})= \begin{cases}1-\exp \left[-\left(\frac{\tilde{h}}{\tilde{H}_{1}}\right)^{k_{1}}\right] & \tilde{h}<\tilde{H}_{t r} \\ 1-\exp \left[-\left(\frac{\tilde{h}}{\tilde{H}_{2}}\right)^{k_{2}}\right] & \tilde{h} \geq \tilde{H}_{t r},\end{cases}$

where $\tilde{H}_{1}=H_{1} / H_{r m s}, \tilde{H}_{2}=H_{2} / H_{r m s}$ and $\tilde{H}_{t r}=H_{t r} / H_{r m s}$ (a tilde above a quantity meaning thus that it was normalized using $H_{r m s}$ ). The distribution function (6) has five unknown parameters $\tilde{H}_{t r}, \tilde{H}_{1}, \tilde{H}_{2}, \kappa_{1}$ and $\kappa_{2}$ :

- On the basis of fits to measurements, Battjes and Groenendijk (2000) fixed the exponents $\kappa_{1}$ and $\kappa_{2}$ as $\kappa_{1}=2$ and $\kappa_{2}=3.6$. Note that the Rayleigh distribution is a particular case of the Weibul distribution when the exponent is equal to 2. Fixing $\kappa_{1}=2$ Battjes and Groenendijk (2000) ensure that their distribution can converge to the Rayleigh distribution when $\tilde{H}_{t r}$ is high enough (no depth effects).

- Also on the basis of fits to measurements, Battjes and Groenendijk (2000) define the transitional wave height as a function of the foreshore slope $\alpha$ and the water depth $d$ :

$$
H_{t r}=(0.35+5.8 \tan \alpha) d \text {. }
$$

- Once $\kappa_{1}, \kappa_{2}$ and $\tilde{H}_{t r}$ are fixed, $\tilde{H}_{1}$ and $\tilde{H}_{2}$ can be computed from the following two constraints. First, in order to have continuity of the distribution function at $\tilde{H}_{t r}$, one needs to impose that

$$
\left(\frac{\tilde{H}_{1}}{\tilde{H}_{t r}}\right)^{\kappa_{1}}=\left(\frac{\tilde{H}_{2}}{\tilde{H}_{t r}}\right)^{\kappa_{2}} .
$$

Second, to assure consistency with the Rayleigh distribution, the second moment of the probability density function has to equal 1 , implying that

$$
\tilde{H}_{r m s}=\sqrt{\tilde{H}_{1}^{2} \gamma\left(\frac{2}{\kappa_{1}}+1,\left(\frac{\tilde{H}_{t r}}{\tilde{H}_{1}}\right)\right)^{\kappa_{1}}+\tilde{H}_{2}^{2} \Gamma\left(\frac{2}{\kappa_{2}}+1,\left(\frac{\tilde{H}_{t r}}{\tilde{H}_{2}}\right)^{\kappa_{2}}\right)}=1,
$$

where $\gamma(a, x)=\int_{0}^{x} e^{-t} t^{a-1} d t$ and $\Gamma(a, x)=\int_{x}^{\infty} e^{-t} t^{a-1} d t$ are the incomplete gamma functions.

Using the model above estimates of $\tilde{H}_{x \%}$ can be obtained as function of $\tilde{H}_{t r}$, as done in Battjes and Groenendijk (2000, Table 2$)^{2}$. As noted by them, for $\tilde{H}_{t r}>2.75$ the estimates of $\tilde{H}_{t r}$ converge to those of the (normalized) Rayleigh distribution. Note also that, according to Eq. (7), and consistently

\footnotetext{
2 The results in Battjes and Groenendijk (2000) were computed using Eq. (9) with the first argument of $\Gamma(a, x)$ taken as $a=2 / 3.5+1$ instead of $a=2 / 3.6+1$.
} 
with the known spatial lag in the process of breaking compared to depth changes, a steeper bottom leads to a higher $H_{t r}$ (smaller deviation of the BG-distribution from the Rayleigh distribution).

Also on the basis of measurements, Battjes and Groenendijk (2000) express the root-mean-square wave height not only as a function of $m_{0}$, as in the case of the Rayleigh distribution, but also as a function of the water depth:

$\frac{H_{r m s}}{\sqrt{m_{0}}}=2.69+3.24 \frac{\sqrt{m_{0}}}{d}$.

Furthermore, as fixed by Battjes and Groenendijk (2000) and contrary to what is the case with the Rayleigh distribution, as $d \rightarrow \infty H_{r m s} / \sqrt{m_{0}} \rightarrow 2.69$, which Battjes and Groenendijk (2000), following Goda (1979), argue to be the valid ratio for sea waves in deep waters with a broad-banded frequency spectrum. Recall that the $H_{r m s} / \sqrt{m_{0}}$ ratio equals $\sqrt{8}=2.83$ when a narrow frequency spectrum is assumed, as in the case of the Rayleigh distribution.

Once $m_{0}, \alpha$ and $d$ are known, $H_{x \%}$ can be computed. Fig. 1 shows, for an illustrative example in which $m_{0}$ was set equal to 1 and the slope to 1:250, the variation of the Battjes and Groenendijk estimates (the dashed black line) of $H_{0.1 \%}$ with the depth.
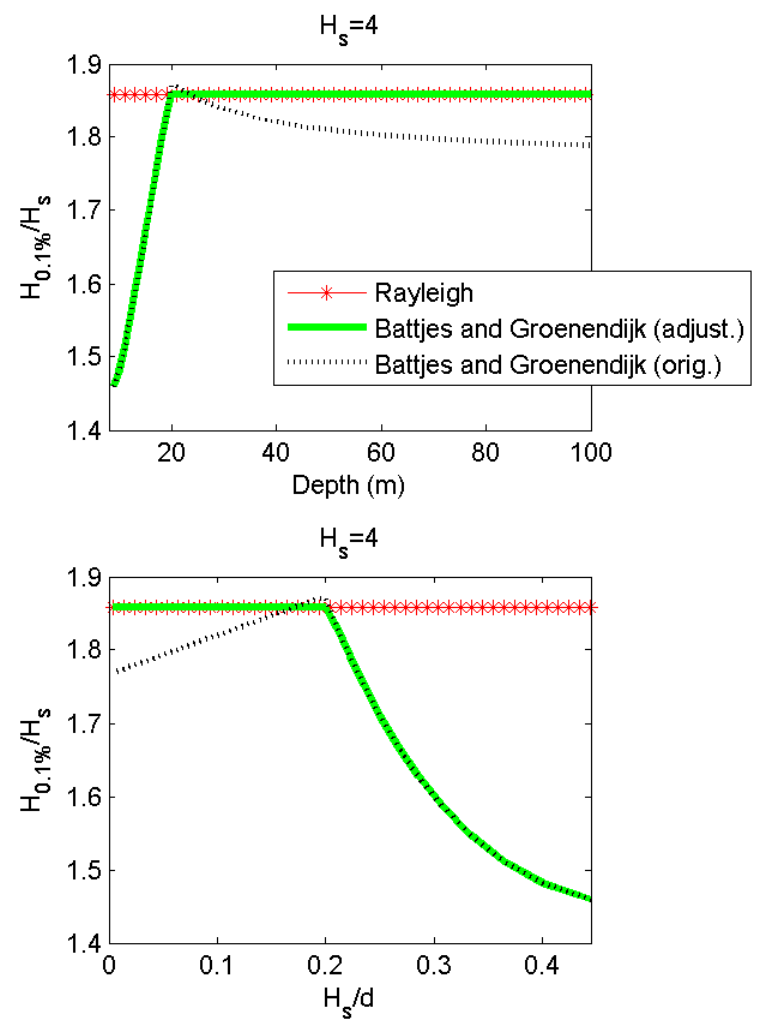

Figure 1. Comparisons between $H_{0.1 \%} / H_{s}$ estimates of the Rayleigh and Battjes and Groenendijk distributions. $H_{s}=4 \mathrm{~m}\left(m_{0}=1\right)$, slope $=1: 250$.

The figure shows that as the depth increases the Battjes and Groenendijk estimates approach those of the Rayleigh, actually overshooting them, and then the Battjes and Groenendijk estimates decrease, converging to a value well below the Rayleigh limiting value (since (10) will converge to 2.69 instead of $\sqrt{8}$ as fixed in (1)). This behavior is undesirable because, assuming the validity of the Rayleigh distribution in deep waters, a shallow water alternative should in principle converge to the Rayleigh distribution in deep waters. This problem does not arise when considering the normalized distributions, since, as noted earlier, the normalized BG-distribution does converge to the normalized 
Rayleigh distribution in deep waters. The problem does arise when in order to obtain the wave height one needs to multiply the normalized wave height by $H_{r m s}$ (cf. Eq. (5)). This 'denormalization' has as consequence that the estimates of $H_{0.1 \%}$ do not show the nice asymptotic behavior of $\tilde{H}_{0.1 \%}$. In order to obtain 'well-behaved' quantile estimates of the BG-distribution, in our implementations of the BGdistribution we have adopted the following procedure: if the estimates of the BG distribution do not exceed those of the Rayleigh and $\tilde{H}_{t r}<2.75$ then we use the former; otherwise, we use the Rayleigh estimates. As can be observed also in Fig. 1, this adjustment ensures that the Battjes and Groenendijk estimates converge to the Rayleigh estimates in deep waters.

Note that the need for this adjustment in the implementation of the BG-distribution is illustrated here using $H_{0.1 \%}, m_{0}=1$ and a slope of $1: 250$, but the problem is obviously the same if other quantiles and values are used.

\section{DESCRIPTION OF THE MEASUREMENTS}

\section{Wave flume data}

The physical model tests have been executed in Deltares' Scheldt Flume. This wave flume has a length of $55 \mathrm{~m}$, a width of $1 \mathrm{~m}$ and a height of $1.2 \mathrm{~m}$. The facility is equipped with a wave board for generating regular/monochromatic and irregular/random waves in relatively shallow-water by a translatory wave board. The on-line computer facilities for wave-board control, data acquisition and data processing allow for direct control and computation of relevant wave characteristics. Wave energy spectra can be prescribed by using standard or non-standard spectral shapes or by prescribing specific time series of wave trains. Second-order wave generation for irregular/random waves is available which produces natural wave trains directly at the wave board by simultaneously generating bound long waves. Furthermore, the wave board has active wave absorption, which prevents reflected waves to re-reflect against the wave board, thereby not disturbing the measurements.

One bathymetrical configuration has been considered under 32 boundary wave conditions. At several positions the wave conditions have been determined with the use of wave gauges. To reduce reflections as much as possible a wave damping beach was placed at the end of the flume which acts as a passive reflection absorber.
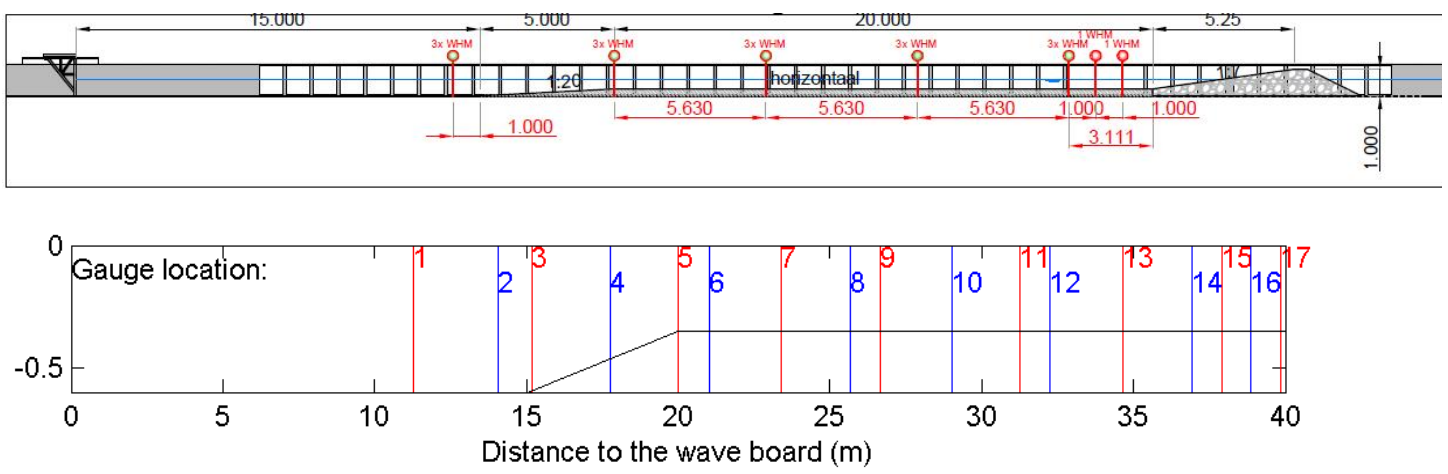

Figure 2. Top panel: Flume test set-up. Bottom panel: Wave gauges approximate location and reference number.

Fig. 2 shows the built in bottom configuration and the location of the wave gauges. The wave board is located at the left end of the figure and the damping beach at the right end of the figure. In front of the wave board the water depth is $0.60 \mathrm{~m}$, at a distance of $15 \mathrm{~m}$ from the wave board the depth is decreased to $0.35 \mathrm{~m}$ in a region with a $1: 20$ slope. After reaching the $0.35 \mathrm{~m}$ region the depth remains constant until the damping beach at the end of the flume. Note that instead of having constant depth bottom in the whole flume, we decided to built a deeper region in front of the wave board in order to eliminate the wave board effects from the data.

We shall in this study concentrate on the individual wave height distributions measured at locations 7 to 15 and in particular in location 14 , which is reached by waves that are already for some wave lengths propagating over the $0.35 \mathrm{~m}$ depth flat bottom (cf. bottom panel of Fig. 2). 


\begin{tabular}{|c|c|c|c|c|c|}
\hline Condition & symbol & $H_{s}(\mathrm{~m})$ & $T_{p}(\mathrm{~s})$ & $H_{s} / d, d=0.35 \mathrm{~m}$ & $s$ \\
\hline $1 \& 17$ & 0 & 0.21 & 7.56 & 0.6 & 0.015 \\
\hline $2 \& 18$ & $\Delta$ & 0.21 & 5.67 & 0.6 & 0.020 \\
\hline $3 \& 19$ & + & 0.21 & 3.78 & 0.6 & 0.031 \\
\hline $4 \& 20$ & T & 0.21 & 2.83 & 0.6 & 0.041 \\
\hline $5 \& 21$ & 0 & 0.18 & 6.30 & 0.5 & 0.015 \\
\hline $6 \& 22$ & $\Delta$ & 0.18 & 4.72 & 0.5 & 0.020 \\
\hline $7 \& 23$ & + & 0.18 & 3.15 & 0.5 & 0.031 \\
\hline $8 \& 24$ & I & 0.18 & 2.36 & 0.5 & 0.042 \\
\hline $9 \& 25$ & 0 & 0.14 & 7.56 & 0.4 & 0.010 \\
\hline $10 \& 26$ & $\Delta$ & 0.14 & 3.78 & 0.4 & 0.020 \\
\hline $11 \& 27$ & + & 0.14 & 2.52 & 0.4 & 0.031 \\
\hline $12 \& 28$ & & 0.14 & 1.89 & 0.4 & 0.043 \\
\hline $13 \& 29$ & 0 & 0.11 & 5.67 & 0.3 & 0.010 \\
\hline $14 \& 30$ & $\Delta$ & 0.11 & 2.83 & 0.3 & 0.021 \\
\hline $15 \& 31$ & + & 0.11 & 1.89 & 0.3 & 0.032 \\
\hline $16 \& 32$ & & 0.11 & 1.42 & 0.3 & 0.045 \\
\hline
\end{tabular}

Table 1 shows for each test the imposed wave conditions ( $H_{s}$ and peak wave period $T_{p}$ ) at the wave board. For tests 1 to 16 JONSWAP spectra with a peak enhancement factor of 1 (i.e. PiersonMoskowitz spectra) have been applied and for tests 17 to 32 JONSWAP spectra with a peak enhancement factor of 3.3 have been applied. All tests have been carried out using approximately 2,000 waves. The variations in the imposed wave conditions are in terms of the significant wave height to water depth ratio and wave steepness $s$ (the ratio between $H_{s}$ and the wave length computed inputting $T_{p}$ and a $0.35 \mathrm{~m}$ depth in the wave dispersion relation). These wave conditions were chosen so that, if they where to reach the 0.35 flat bottom region unchanged, $H_{s} / d$ would take values between 0.3 and 0.6 and the wave steepness would take values between 0.010 and 0.045 (cf. Table 1). However, the wave conditions do not remain unchanged as can be seen in Table 2 that shows the measured conditions at location 14. The $H_{s} / d$ ratios at location 14 do not exceed 0.46 and the wave steepness takes values between 0.010 and 0.041 and the number of waves varies between 1,731 and 4,512 waves. 


\begin{tabular}{|c|c|c|c|c|c|}
\hline Cond. & $H_{s}(\mathrm{~m})$ & $T_{p}(\mathrm{~s})$ & $H_{s} / d$ & $s$ & $N$ \\
\hline 1 & 0.15 & 8.19 & 0.43 & 0.010 & 4322 \\
\hline 2 & 0.15 & 6.31 & 0.43 & 0.013 & 3639 \\
\hline 3 & 0.16 & 3.53 & 0.46 & 0.025 & 2583 \\
\hline 4 & 0.16 & 3.42 & 0.46 & 0.026 & 2023 \\
\hline 5 & 0.15 & 6.83 & 0.42 & 0.012 & 3866 \\
\hline 6 & 0.15 & 5.14 & 0.43 & 0.016 & 3158 \\
\hline 7 & 0.16 & 3.53 & 0.45 & 0.024 & 2252 \\
\hline 8 & 0.15 & 2.64 & 0.44 & 0.032 & 1765 \\
\hline 9 & 0.13 & 8.19 & 0.37 & 0.009 & 4243 \\
\hline 10 & 0.14 & 2.26 & 0.41 & 0.036 & 2492 \\
\hline 11 & 0.14 & 2.80 & 0.39 & 0.027 & 1877 \\
\hline 12 & 0.13 & 2.18 & 0.38 & 0.034 & 1876 \\
\hline 13 & 0.12 & 6.31 & 0.33 & 0.010 & 3206 \\
\hline 14 & 0.11 & 3.16 & 0.32 & 0.020 & 1981 \\
\hline 15 & 0.10 & 2.18 & 0.30 & 0.027 & 1939 \\
\hline 16 & 0.09 & 1.48 & 0.27 & 0.038 & 1844 \\
\hline 17 & 0.15 & 7.46 & 0.42 & 0.011 & 4512 \\
\hline 18 & 0.15 & 5.82 & 0.42 & 0.014 & 3992 \\
\hline 19 & 0.16 & 3.88 & 0.45 & 0.022 & 2794 \\
\hline 20 & 0.16 & 3.02 & 0.45 & 0.029 & 2197 \\
\hline 21 & 0.14 & 6.28 & 0.40 & 0.012 & 4148 \\
\hline 22 & 0.14 & 4.80 & 0.41 & 0.016 & 3333 \\
\hline 23 & 0.15 & 3.26 & 0.44 & 0.026 & 2446 \\
\hline 24 & 0.15 & 2.40 & 0.44 & 0.036 & 2093 \\
\hline 25 & 0.13 & 7.46 & 0.37 & 0.009 & 4467 \\
\hline 26 & 0.14 & 3.88 & 0.41 & 0.020 & 2628 \\
\hline 27 & 0.14 & 2.64 & 0.39 & 0.029 & 2162 \\
\hline 28 & 0.14 & 2.14 & 0.39 & 0.036 & 1950 \\
\hline 29 & 0.11 & 5.82 & 0.32 & 0.011 & 3421 \\
\hline 30 & 0.11 & 2.91 & 0.32 & 0.021 & 2072 \\
\hline 31 & 0.11 & 1.94 & 0.31 & 0.032 & 2011 \\
\hline 32 & 0.10 & 1.46 & 0.28 & 0.041 & 1731 \\
\hline
\end{tabular}

\section{Field data}

The field data considered in this study were measured in the lakes IJssel and Sloten in The Netherlands, at measuring locations F2 and SL29, respectively, see Fig. 3. Both lakes are shallow and have a fairly flat, sandy bottom.

The wave and wind data for these lakes have been extensively validated and analysed by Bottema (2007), further by Bottema and Van Vledder (2009) and by Van der Westhuysen (2010) to study depth-induced wave breaking under finite-depth wave growth conditions in flat bottoms. For this analysis, the same storm data from the SW-W wind direction sector recorded at station FL2 in Lake IJssel and station SL29 in Lake Sloten in the period 2002-2007 used in the above mentioned publications is considered. All measurement data are supplied on the DVD which is enclosed in Bottema (2007). From that DVD the sea surface elevation and water level observations for the following days 22/2/2002, 27/10/2002, 08/01/2005, 12/2/2005 and 18/1/2007 in the FL2 location and the following days: 12/2/2002, 26/2/2002; 27/10/2002; 20/2/2004 and 18/01/2007 in the SL29 location were analyzed. The data are available continuously every $0.25 \mathrm{~s}$ in the selected days in files with 20-minutes records each (72 files per day). 


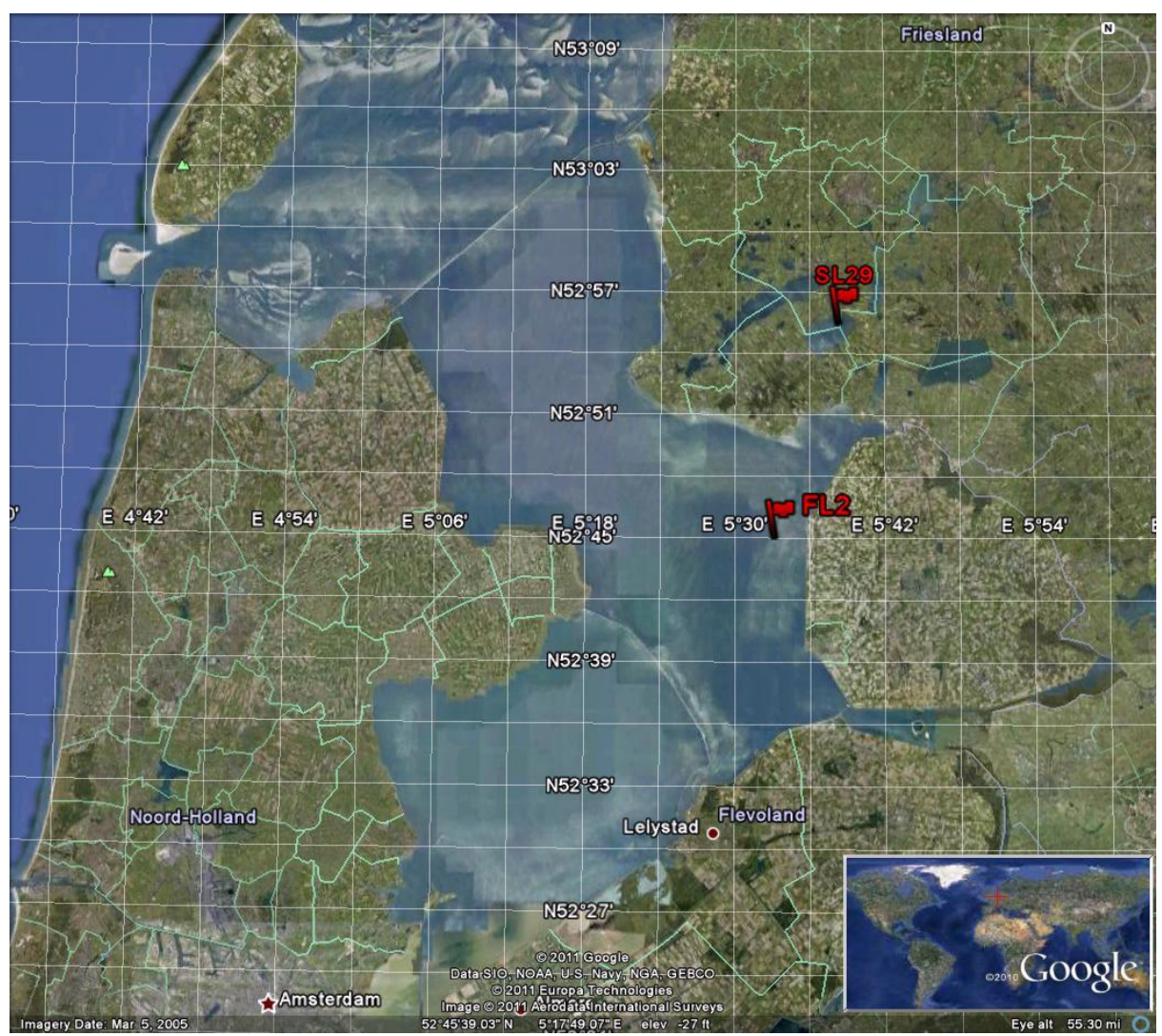

Figure 3. Google earth aerial view of the FL2 and SL29 measuring locations.

\section{DATA ANALYSIS}

In order to check the validity of the BG-distribution we compare the measured $H_{0.1 \%}$ (flume data) or $H_{1 / N}$ (lakes IJssel and Sloten data) with the corresponding Battjes and Groenendijk and Rayleigh estimates. In all cases we use a slope of 1:250 in the BG-distribution (the flatter slope in the data used to calibrate the distribution).

In all cases time series of the surface elevation with a time resolution of 0.25 seconds were available. The signals were analyzed using time (zero down-crossing) and frequency domain analysis. All the $H_{s}$ values presented were computed from the wave spectra, integrating between 0.02 and 1.5 $\mathrm{Hz}$ in the case of flume data and between 0.03 and $1 \mathrm{~Hz}$ in the case of field data. The quantiles were computed directly from the individual wave height data obtained from the zero down-crossing analysis. $H_{0.1 \%}$ was computed by linearly interpolating, when needed, the adjacent empirical quantiles. $H_{1 / N}$ was set equal to the highest individual wave height in the considered time series.

In order to quantify the agreement between the measured and estimated quantiles, we have computed the relative bias,

$e=\frac{y-x}{x} \times 100 \%$,

and the linear slope (with no intercept),

$r=\sum_{i=1}^{n} x_{i} y_{i} / \sum_{i=1}^{n} x_{i}^{2}$,

where $\mathrm{x}$ and $\mathrm{y}$ are respectively the measured value and the value estimated using the distribution.

\section{Wave flume data}

Figure 4 compares the measured and the Battjes and Groenendijk and Rayleigh estimates of $H_{0.1 \%}$ at the flume location 14. The symbols association with the conditions is as indicated in Table 1. 
The blue symbols refer to conditions 1-16 and the red symbols to conditions 17-34. The plot also shows the line with slope $r$ (dashed line).
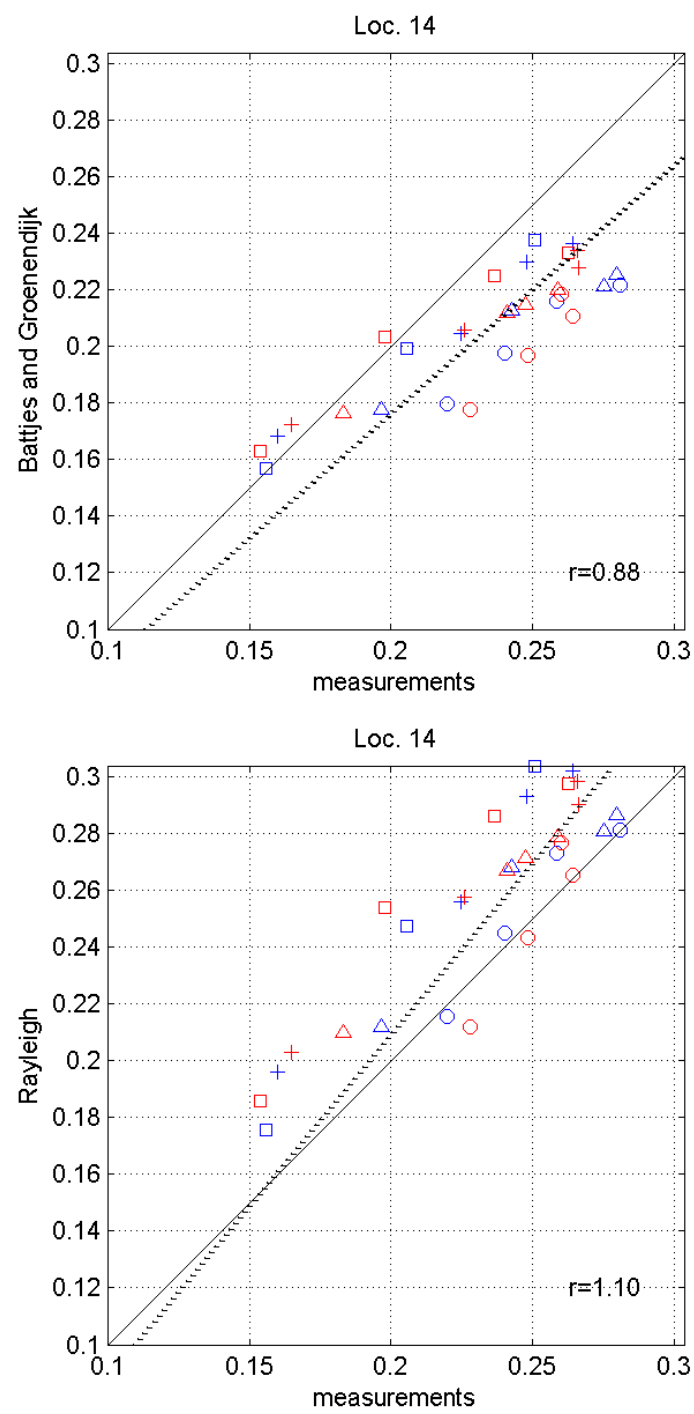

Figure 4. Top panel: Comparison between the measured and the Battjes and Groenendijk estimates of $H_{0.1 \%}$ at the flume location 14. Bottom panel: Comparison between the measured and the Rayleigh estimates of $H_{0.1 \%}$. at the flume location 14. $r$ is the slope of the dashed lines. The used symbols are associated with the conditions as indicated in Table 1. The blue symbols refer to conditions 1-16 and the red symbols to conditions 17-34.

The linear slope in the comparisons with Battjes and Groenendijk estimates is 0.88 , indicating a $12 \%$ underestimation of $H_{0.1 \%}$ (the relative biases reaching values as low as $22 \%$ ), and in the comparisons with the Rayleigh estimates it is 1.1, indicating a $10 \%$ overestimation. Table 3 shows the linear slope between the measurements and Battjes and Groenendijk estimates of $H_{0.1 \%}$ at all locations in the $0.35 \mathrm{~m}$ depth region of the flume and when considering the test in which a JONSWAP and a Pierson-Moskowitz spectra were used separately. The observed underestimation of $H_{0.1 \%}$ by the BG-distribution seems to be independent of the spectral form used.

We have examined the relation between the relative error of the Battjes and Groenendijk estimates and several parameters such as the $H_{s}$ to depth ratio and the steepness and found that there is a strong relation between the wave steepness and the relative bias of the Battjes and Groenendijk estimates; see Figure 5. The figure shows that the steeper the waves the less the underestimation by the BG-distribution. Note also that the lower the steepness the higher the reflection from the wave damping beach, which in the tests was at most of $44 \%$. 


\begin{tabular}{|c|c|c|c|}
\hline Location & All conditions & $\begin{array}{c}\text { Conditions 1-16 } \\
\text { (Pierson-Moskowitz) }\end{array}$ & $\begin{array}{c}\text { Conditions 17-32 } \\
\text { (JONSWAP) }\end{array}$ \\
\hline 7 & 0.82 & 0.83 & 0.82 \\
\hline 8 & 0.83 & 0.84 & 0.83 \\
\hline 9 & 0.84 & 0.84 & 0.83 \\
\hline 10 & 0.86 & 0.86 & 0.86 \\
\hline 11 & 0.86 & 0.85 & 0.86 \\
\hline 12 & 0.87 & 0.86 & 0.87 \\
\hline 13 & 0.87 & 0.87 & 0.87 \\
\hline 14 & 0.88 & 0.88 & 0.88 \\
\hline 15 & 0.89 & 0.89 & 0.89 \\
\hline 16 & 0.90 & 0.88 & 0.91 \\
\hline 17 & 0.89 & 0.88 & 0.91 \\
\hline
\end{tabular}

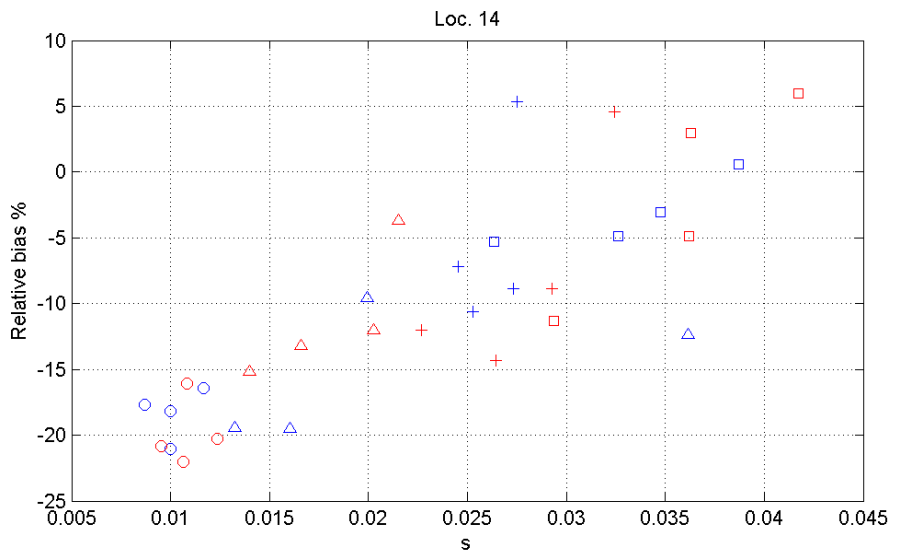

Figure 5. Relation between the relative error of the Battjes and Groenendijk estimates of $H_{0.1 \%}$ and the wave steepness at location 14.

\section{Lake IJssel data}

From all FL2 20-min measurements available in the dates considered (22/2/2002, 27/10/2002, $08 / 01 / 2005,12 / 2 / 2005$ and 18/1/2007) the measurements for which the ratio between $H_{s}$ and the total water depth exceed 0.3 are considered further. In total 44 20-min records fulfill this criterion. For these data the wind speed varies between 17 and $24 \mathrm{~m} / \mathrm{s}$, the wind direction between 210 and 280 ${ }^{\circ} \mathrm{N}$, the water depth between 4 and $5.6 \mathrm{~m}$ and the number of waves between 280 and 380 waves.

Given that the number of wave is less than 1,000 , for these data we analyse the performance of the BG-distribution in terms of $H_{1 / N}$ instead of $H_{0.1 \%}$.

Fig. 6 compares the measured and the Battjes and Groenendijk and Rayleigh estimates of $H_{1 / N}$. The linear slope in the comparisons with Battjes and Groenendijk estimates is 0.93 , indicating a $7 \%$ underestimation of $H_{1 / N}$ (the relative biases reaching values as low as 17\%, cf. Fig. 7), and in the comparisons with the Rayleigh estimates it is 1.07 , indicating a $7 \%$ overestimation.

If 60 -min records are considered instead of the 20 -min records, then the slope between the data and the Battjes and Groenendijk and Rayleigh $H_{1 / N}$ estimates is 0.91 and 1.08, respectively.

Fig. 7 shows the variation of the relative error of the Battjes and Groenendijk estimates (from the 20-min records) with the wave steepness. As was the case with the flume data, although not so clearly seen in this data, there seems to be that the steeper the waves the less the underestimation by the BGdistribution. 

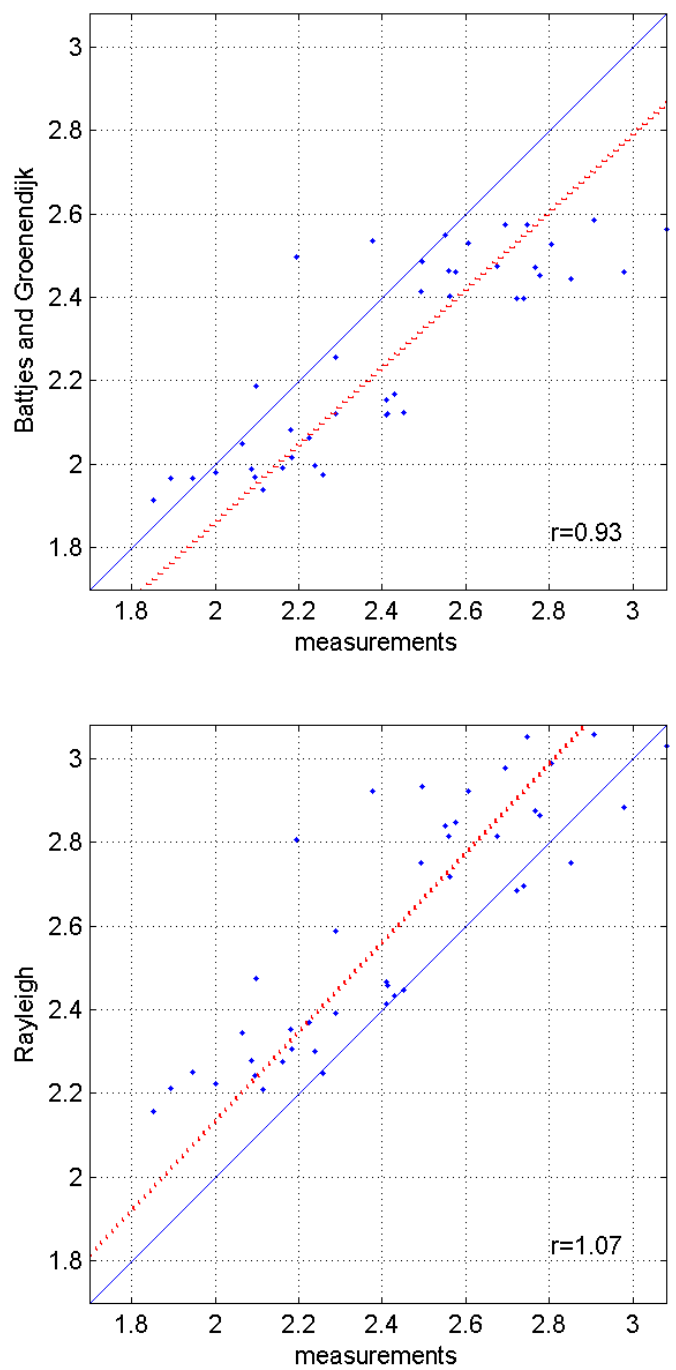

Figure 6. Top panel: Comparison between the measured and the Battjes and Groenendijk estimates of $H_{1 / N}$ at FL2. Bottom panel: Comparison between the measured and the Rayleigh estimates of $H_{1 / N}$ at FL2. N is the number of waves in each 20 -min record. $r$ is the slope of the dashed lines.

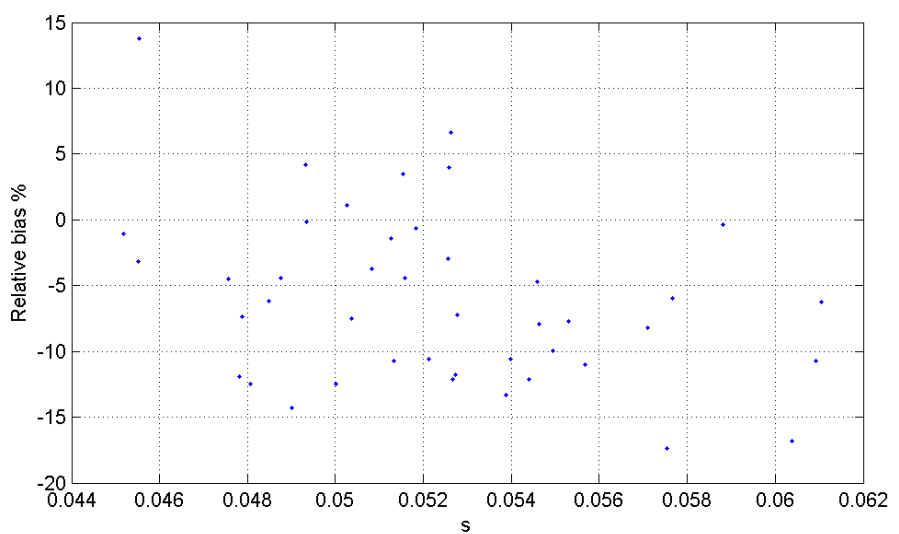

Figure 7. Relation between the relative error of the Battjes and Groenendijk estimates of $H_{1 / N}$ and the wave steepness at location FL2. 


\section{Lake Sloten data}

From all SL29 20-min measurements available in the dates considerer (12/2/2002, 26/2/2002, 27/10/2002, 20/2/2004 and 18/01/2007) the measurements for which the ratio between $H_{s}$ and the total water depth exceed 0.3 are considered further. In total 217 20-min records fulfill this criterion. For these data the wind speed varies between 11 and $23 \mathrm{~m} / \mathrm{s}$, the wind direction between 180 and 300 ${ }^{\circ} \mathrm{N}$, the water depth between 0.9 and $1.6 \mathrm{~m}$ and the number of waves between 430 and 670 waves.

Fig. 8 compares the measured and the Battjes and Groenendijk and Rayleigh estimates of $H_{1 / N}$. The linear slope in the comparisons with Battjes and Groenendijk estimates is 0.85 , indicating a $15 \%$ underestimation of $H_{1 / N}$ (the relative biases reaching values as low as 42\%, cf. Fig. 10), and in the comparisons with the Rayleigh estimates it is 1.05 , indicating a $7 \%$ overestimation.

If 60-min records are considered instead of the 20-min records, then the slope between the data and the Battjes and Groenendijk and Rayleigh $H_{1 / N}$ estimates is 0.84 and 1.07, respectively.

Fig. 9 shows the variation of the relative error of the Battjes and Groenendijk estimates (from the 20-min records) with the wave steepness. Contrary to what was the case with the flume data, there seems to be no relation between the wave steepness and the underestimation by the BG-distribution.
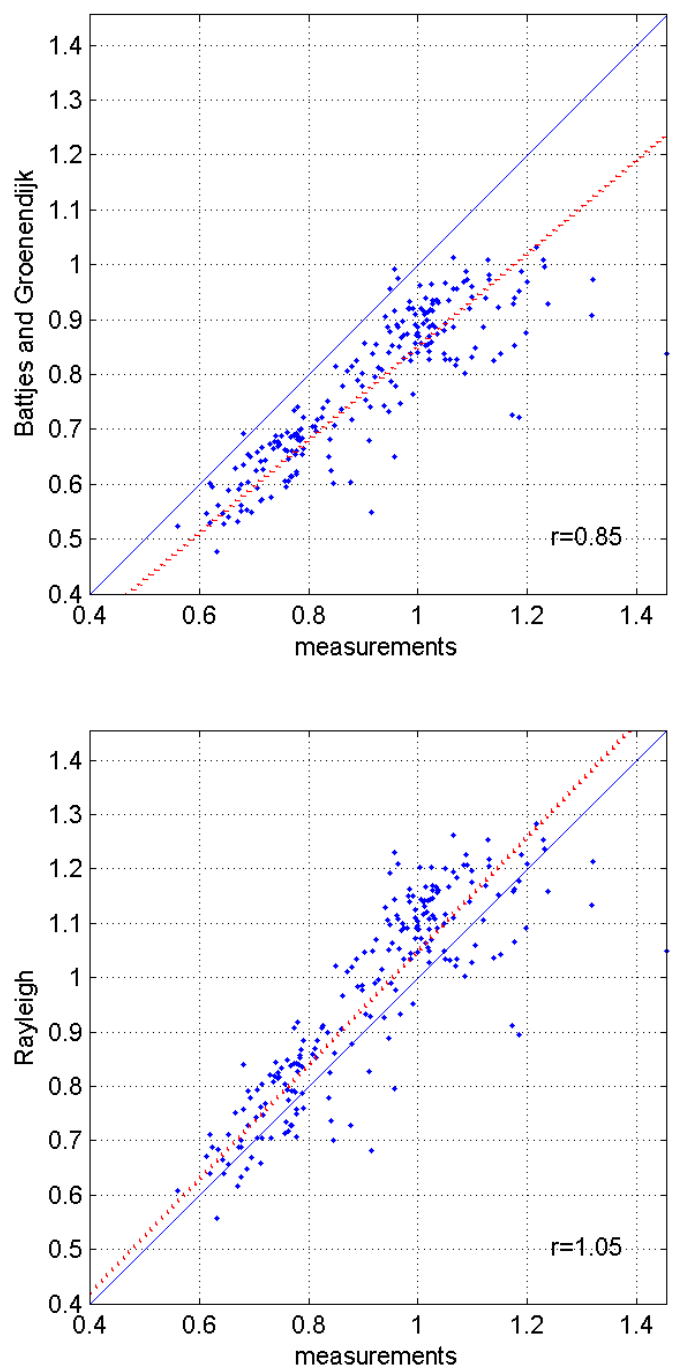

Figure 8. Top panel: Comparison between the measured and the Battjes and Groenendijk estimates of $H_{1 / N}$ at SL29. Bottom panel: Comparison between the measured and the Rayleigh estimates of $H_{1 / N}$ SL29. $\mathbf{N}$ is the number of waves in each 20-min record. $r$ is the slope of the dashed red lines. 


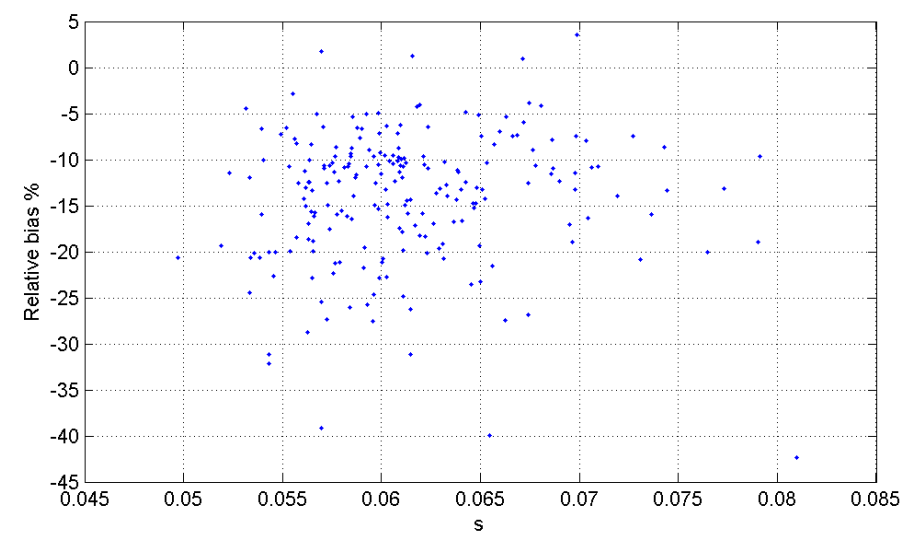

Figure 9. Relation between the relative error of the Battjes and Groenendijk estimates of $H_{1 / N}$ and the wave steepness at location SL29.

\section{Discussion}

In the results shown above we tried to see how the BG-distribution estimates of high quantiles of wave height perform under flat bottom conditions. Recall that the BG-distribution was obtained using experimental data from flumes with foreshore slopes varying from 1:20 to 1:250, and that therefore it is in principle not valid under flat bottom conditions. Nevertheless, we tried to see whether applying the BG-distribution with the flattest slope considered, of 1:250, would still lead to reasonable results. Unfortunately, our results show that the BG-distribution underestimates the high quantiles of wave height under flat bottom conditions. In fact, considering a steeper slope (instead of the flattest) would probably lead to better estimates, since the steeper the slope the closer the estimates are to those of the Rayleigh distribution and since the Rayleigh distribution estimates compare generally better with the measurements than do the BG-distribution estimates.

The results obtained are in line with our expectations since on flat bottoms the ratio of significant wave height to depth has also been found to be higher than one would expect by decreasing the bottom slope in the available formulations for depth-induced wave breaking (Van der Westhuysen, 2010).

Given that the available formulations for depth-induced wave breaking behaved better when not only the relative depth but also the wave steepness was taken into account, Van der Westhuysen (2010) concluded that the modeling of depth-induced wave breaking should be done as function of the non-linearity of the waves in finite depths. He has therefore suggested a formulation for depth-induced wave breaking based on the local shallow water non-linearity, expressed in terms of the biphase of the self-interactions of the spectral peak. His model, in which the biphase is a function of the parametric Ursell number (depending on Hs, the mean wave period and the depth), leads to good results for both flat and steep slopes. Note that, at least in our flume results, the relative errors of the BG-distribution seem to depend also on the wave steepness and thus on the local shallow water non-linearity. The results of Westhuysen (2010) suggest thus that making $H_{t r}$ a function of the biphase instead of the bottom slope may lead to a BG-distribution that is also valid in flat bottom conditions.

Given that the non-linearity of the waves is the important factor defining the high quantiles of wave height, an approach such as that proposed by Janssen and Bidlot (2009) and used to compute the maximal wave height in the wave model of the European Centre for Medium-Range Weather Forecast (ECMWF) should also be feasible to obtain estimates of high wave height quantiles in finite depth regions under flat bottom (and other) conditions. In the ECMWF wave model, the kurtosis of the wave field is computed from the wave spectra as proposed by Janssen (2009) and the wave height distribution (again a modification of the Rayleigh distribution) is defined as a function of the kurtosis. Although the model has been applied and verified mostly in deep waters, the proposed method is general and should also perform well in shallow regions.

\section{CONCLUSIONS AND RECOMMENDATIONS}

The validity of the BG-distribution in constant and finite depth regions was studied using data from tailored tests carried out in Deltares' Scheldt Flume and field data from Lake IJssel and Lake Sloten. 
The comparisons between the quantiles obtained by the measurements and those obtained from the BG-distribution show that the latter generally underestimate the actual high wave heights in regions with shallow flat bottoms (i.e. outside its range of validity). The mean underestimations range between 7 and 15\% when a slope of 1:250 is applied in the BG-distribution.

Note that the flume and field data differ in terms of wind input. The flume data have no active wind input and the field data come from storm periods with active winds in the flat bottom region. Nevertheless, in both, higher individual wave heights are attained than in the shallow (1:250 slope) foreshore situation of the BG-distribution.

Given that the BG-distribution estimates under flat bottom conditions are on average nonconservative, in current metocean studies we recommend that an on average conservative approach be taken and that in constant and finite depths the Rayleigh distribution be used instead of the BGdistribution to estimate the high quantiles of wave height.

We recommend also that reliable estimates of high quantiles under flat bottom conditions be sought. In our opinion this can be done following at least two approaches. One approach would be to extend the BG-distribution in order that it also performs well under flat bottom conditions. An extension along the same lines as the one proposed by Van der Westhuysen (2010), in which the wave dissipation is modelled in terms of the parametric biphase of the self-interactions of the spectral peak instead of the bottom slope, seems promising. Such a reformulation of the BG-distribution should also deal with overshooting problems of the present formulation (cf. Fig. 1) and its estimates of quantiles should converge to the Rayleigh estimates in deep waters. Another approach would be to estimate the wave kurtosis from the wave spectra as proposed by Janssen (2009) and define the wave height distribution as a function of the kurtosis (again a modification of the Rayleigh distribution); see Janssen and Bidlot (2009). Note that both approaches require more information from the wave spectrum than just $m_{0}$, but spectral information is often available since metocean studies are usually based on spectral models results.

\section{ACKNOWLEDGEMENTS}

The authors are grateful to Mr. Ivo van der Werf and Mr. John Coolegem for their efforts in carrying out the flume experiments.

\section{REFERENCES}

Battjes, J.A., and H.W. Groenendijk. 2000. Wave height distributions on shallow foreshores, Coastal Engineering, 40, 61-182.

Bottema, M. 2007. Measured wind-wave climatology Lake IJssel (NL). Main results for the period 1997-2006, Report RWS RIZA 2007.020, July 2007.

Bottema, M., and G.P. van Vledder. 2009. A ten-year data set for fetch- and depth-limited wave growth, Coastal Engineering, 56, doi:10.1016/j.coastaleng.2009.01.012.

Goda, Y. 1979. A review on statistical interpretation of wave data, In: Report of the Port and Harbour Research Institute, Japan, 18, 5-32.

Goda, Y. 1985. Random seas and design of maritime structures, University of Tokyo Press.

Holthuijsen, L.H. 2007. Waves in oceanic and coastal waters, Cambridge University Press.

Janssen, P. A. E M. 2009. On some consequences of the canonical transformation in the Hamiltonian theory of water waves, J. Fluid Mech., 637, 1-44.

Janssen, P. A. E. M. and J.-R. Bidlot. 2009. On the extension of the freak wave warning system and its verification, Technical Report 588, European Centre for Medium-rangeWeather Forecasting.

Longuet-Higgins, M.S. 1952. On the statistical distribution of the heights of sea waves, J. Mar. Research, 11, 385-398.

Rattanapitikon, W. 2010. Verification of conversion formulas for computing representative wave heights, Ocean Engineering, 37, 1554-1563.

Van der Westhuysen, A.J. 2010. Modelling of depth-induced wave breaking under finite-depth wave growth conditions, J. Geophys. Res., 115, C01008, doi:10.1029/2009JC005433. 